\title{
Sensory and adaptive access of manufacturing equipment resources in cloud manufacturing
}

\author{
LIU Mingli ${ }^{1, a}$, LIU Yongbo ${ }^{2}$ \\ 1 Liaoning Institute of Science and TechnologyBenxi, Liaoning, China \\ 2 Liaoning Institute of Science and TechnologyBenxi, Liaoning, China
}

\begin{abstract}
Sensory and adaptive access of manufacturing equipment (ME) is the key of physical resources virtualization in cloud manufacturing (CM). Three aspects including status sensory of ME, Internet of Things (IoT) and adaptive access of $\mathrm{CM}$ service platform were elaborated to analyze the characteristics and to investigate the related technologies. The importance of fiber Bragg grating sensing technology for ME sensory was analyzed. The heterogeneous fusion system and fusion access method as well as device of equipment resources were discussed. The access of ME resources for CM service platform was investigated based on three aspects including information fusion, description and retrieval and service release. Taking the realization of intelligent sensory and adaptive access for typical ME resources as an example, the prototype system, the sensory of ME resources and the realization of IoT of $\mathrm{ME}$ resources as well as the adaptive access method for CM service platform were expounded.
\end{abstract}

\section{Introduction}

Cloud manufacturing $(\mathrm{CM})$ is a new kind of manufacturing method and technology which is serviceoriented, efficient, low consumption, and is based on the networked and agile knowledge, and it will promote the manufacturing to be agile, green and intelligent[1-2]. In $\mathrm{CM}$ architecture, the physical resources layer is at the bottom of the architecture and is the base of acquiring information of manufacturing resources and realizing management and invocation of $\mathrm{CM}$ resources service, and it is also the premise of interaction of resources information in CM[3-4].

Physical resources layer's main task is to make all kinds of physical manufacturing resources access to the network to realize comprehensive interconnection and sharing of physical resources in $\mathrm{CM}$, and to provide the interface of virtual encapsulation and service invocation of $\mathrm{CM}$ resources through a variety of advanced information and communication technologies. Sensory and access of physical manufacturing resources is the key to realize full sharing and seamless collaboration of equipment resources, especially in $\mathrm{CM}$ system for group enterprises. As the core component of physical manufacturing resources in $\mathrm{CM}$, sensory and adaptive access of ME need urgent research.

In $\mathrm{CM}$ system, ME resources are the most significant manufacturing ability. Sensory and adaptive access technology can support for the virtual encapsulation, scheduling and optimal allocation of manufacturing resources, so as to improve the utilization and production efficiency of $\mathrm{CM}$ resources in the form of optimal dynamic production scheduling and to eliminate all kinds of faults promptly in production, and thus improving processing quality and reducing cost.

This paper conducts a comprehensive analysis of sensory and adaptive access technology of $\mathrm{CM}$ equipment resources from three aspects, including sensory of ME resources, IoT and adaptive access for CM service platform, and some specific applications are given to further explain the key implementation technology and method.

\section{Sensory of manufacturing equipment resources}

Sensory of ME resources is the precondition and base to realize virtualization of $\mathrm{CM}$ resources. Diversification, large-scale and high precision of ME, and high speed, large load, large displacement and other abnormal conditions during processing, and rugged environment, make sensory of ME resources face enormous challenges.

\subsection{Characteristics of sensory of manufacturing equipment resources}

Sensory of ME resources in CM mainly has the following characteristics:

(1) Rugged and complex environment

The changeable environment, complex structure of ME, precision in processing, small volume and light weight of sensors, and the existence of strong magnetic field and

\footnotetext{
$\overline{{ }^{a} \text { Corresponding author: mumoulml } @ 163 . c o m ~}$
} 
high voltage, bring many great challenges to the sensory of ME.

(2) Massive and various sensory data

1) Massiveness

All kinds of $\mathrm{ME}$ are used in production process, and each of these equipment may need to sense multiple parameters, thus the produced data is staggering.

2) Polymorphism

The large-scale of ME makes different equipment and even the same equipment sense different status parameters in different ways, thus causing the sensory data of multi-source and polymorphism.

3) Relevance

Different sensory data serve together in the manufacturing process, and they inevitably have relevance. Description data of the same entity are relevant in time. Description data of different entities are relevant in space. Description data of entity's different dimensions are also relevant.

(3) Different sensory demand

Sensory demand of ME varies for different application requirements. It is mainly reflected in the real-time, accuracy, reliability, security and compatibility of sensory.

(4) Coexisting of various sensory methods

There are many types of ME in manufacturing process, and sensory parameters differ in face of different application demands, thus leading to different sensory methods.

\subsection{Manufacturing equipment status sensory based on fiber Bragg grating sensing}

In order to obtain real-time operation status information of $\mathrm{ME}$ during processing and to eliminate all kinds of faults in operation timely, there need the support of the effective sensing technology and method. All kinds of sensors such as torque, acceleration, temperature, image and displacement sensors can generally be installed in the equipment to get the running status data and to monitor the equipment operation. But because of the complicated and rugged manufacturing environment and the various limitations of traditional sensing technology, many physical parameters cannot be measured, especially the dynamic, real-time and online monitoring. Fiber Bragg grating sensing technology has the characteristics of one line with multipoint and none source with multi field. And owing to its unique performance, the fiber Bragg grating sensing is a perfect method to realize long-term and real-time status sensory and monitoring and information acquisition of large equipment and large CNC machine tools during their operation[5].

In order to implement the comprehensive sensory of $\mathrm{ME}$, there need to study the layout plan of measurement points of sensors, and to study the multi field coupling, multi parameters, real-time and dynamic online status sensory method. At the same time, develop the embedded, high precision, and high speed wavelength demodulation of fiber Bragg grating sensors. Through the mechanical status analysis of the complex interface of structure, fiber, elastomer and composite material to study the tool's strain transfer problem in processing and to realize the sensitive sensory of ME in operating status based on the strain transfer efficiency.

Embedded information processing communication system can acquire and process multi-source, polymorphic and massive sensory information of $\mathrm{ME}$, and thus realizing the real-time, dynamic and intelligent sensory of ME running status.

\section{3 loT of manufacturing equipment resources}

Sensory of various ME resources status information in $\mathrm{CM}$ will necessarily come into a heterogeneous network system with multiple access methods, multiple transmission rates and various service quality requirements. Terminals can be access to the heterogeneous network by wired or wireless ways(such as bluetooth, $802.15 \mathrm{x}, 802.11 \mathrm{x}$, mobile cellular networks, LAN cable, etc.) and the network coverage, bandwidth and Quality of Service(QoS) differ[6]. How to make heterogeneous networks harmonize and cooperate with each other, and how to realize the efficient IoT of ME resources in an optimized way, is another key problem need to be solved about sensory and adaptive access in $\mathrm{CM}$ equipment resources.

\section{1 loT system of manufacturing equipment resources}

The IoT of ME resources is main to realize the connection and sensory of various ME resources and transmission of acquired information. Different ME use different communication devices and technologies to sense and acquire information, such as fiber sensing technology is adopted to intelligently sense the complex running status of ME. The massive and high real-time data can be access to the IoT of manufacturing environment by means of cable. And some CNC machine tools have its own monitoring modules to acquire and output status information through embedded adaptive devices.

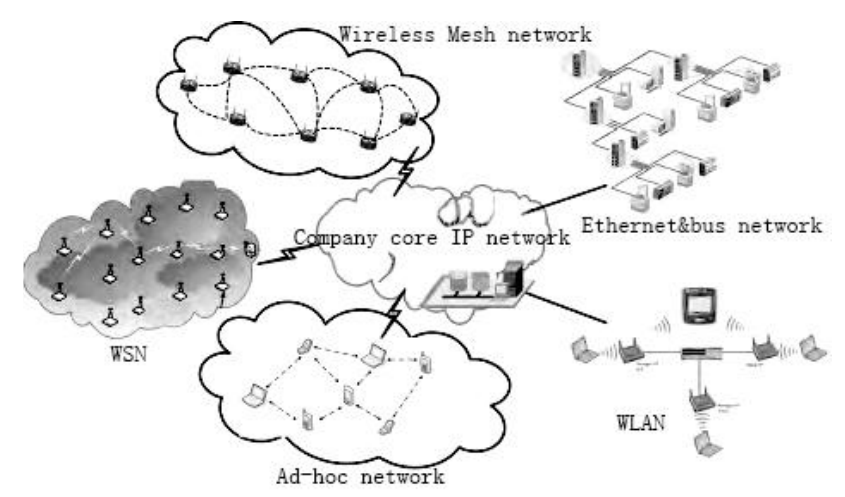

Figure 1. Heterogeneous network for IoT of manufacturing equipment. 
In the heterogeneous network, there are existing Ethernet, bus network, wireless sensor network, wireless Mesh network and many other different types of networks, and different types of networks use different communication technologies respectively[7], as shown in figure 1 .

\subsection{Key technologies of IoT of manufacturing equipment resources}

According to the characteristics of status sensory of different $\mathrm{ME}$ and data transmission requirements, various communication technologies are used comprehensively to realize the combination of wired and wireless, combination of broadband and narrowband, combination of sensory network and communication network thus providing a good network support for the acquisition, encapsulation and invocation of $\mathrm{ME}$ resources. The following aspects of tasks are studied to realize the comprehensive IoT of $\mathrm{ME}$ resources and to solve the seamless fusion of various heterogeneous networks:

(1) Choose reasonable adaptive access method in view of the different ways of sensory and different requirements of sensory data transmission.

(2) Design reasonable network architecture and network interface and make full use of existing network infrastructure to complement all kinds of networks.

(3) Select reasonable structure mode of heterogeneous network[8].

(4) Apply appropriate adaptive access technology of heterogeneous network[9].

Wireless communication in industrial also calls for the attention of IoT of ME resources. The wireless network in manufacturing environment has the characteristics of large scale and complex environment and it is vulnerable to strong noise produced by $\mathrm{ME}$, other narrowband or broadband interference produced by wireless devices and multiplicity interference of signal transmission caused by moving people or equipment reflection.

In addition, in a service-oriented, scalable and intelligent $\mathrm{CM}$ service platform the physical sensory information may be stolen, tampered or destroyed during transmission. At the same time, during the sensory of $\mathrm{ME}$ resources it might be infected by virus. So the safety of sensory information transmission and the security of CM equipment itself should also be paid attention.

\section{Adaptive access of manufacturing equipment resources for $\mathrm{CM}$ service platform}

The adaptive access of $\mathrm{ME}$ resources for $\mathrm{CM}$ service platform mainly studies the intelligent analysis and preprocessing of sensory information of manufacturing resources. Starting from the basic principle of data fusion and making full use of multi-dimensional information of manufacturing resources we explore the correlation of all kinds of information thus providing the foundation for the users to effectively use the dispersed manufacturing resources in $\mathrm{CM}$.

\subsection{Sensory information fusion}

According to the characteristics of the sensory data, information fusion method can be used to build multilayer fusion model to analyze and preprocess the huge amounts of information[10]. The fusion architecture can be divided into model layer, characteristic layer, processing layer and decision layer, and the four layers complete the tasks of modeling, feature extraction and model matching, conversion processing and real-time storage, rules decision and sensory information analysis respectively. The information flow and data flow of each layer are combined in the fusion architecture. And according to the characteristics and purposes of sensory information sources, the flow level and handling method of information are intelligently decided so as to balance speed, data quantity and application. Sensory information fusion model is shown in figure 2 .

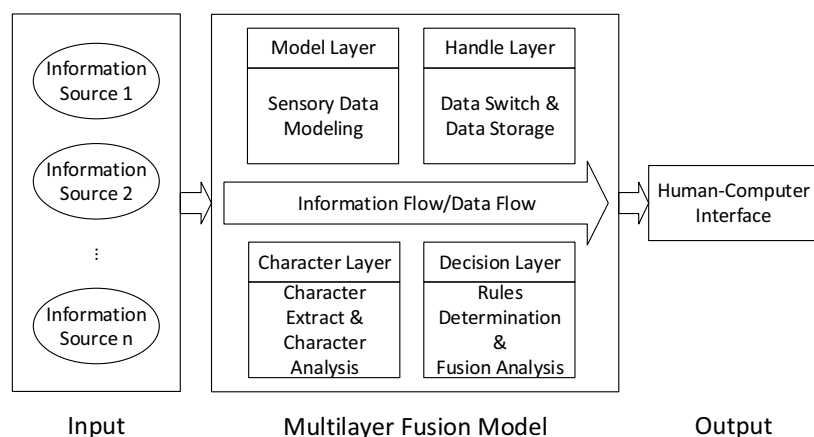

Figure 2. Sensory information fusion model.

\subsection{Description and intelligent retrieval of sensory information}

Description and intelligent retrieval of sensory information is the key to realize the users' full use of distributed ME resources in CM. In this paper, the method of ontology is used to build the ontology model of ME resources to publicly and unitedly describe its concepts, objects, procedures, rules and targets in digital and denote it in ontology description language. The built model are connected with structure, function, property and knowledge of equipment resources to provide data and information to achieve effective and intelligent searching and matching of equipment resources[11]. In addition, the middleware technology is adopted to encapsulate and release equipment resources service to realize sharing of information in different regions and different platforms.

\section{Example of prototype system}

The prototype system composition is shown in figure 3, including status sensory of ME resources, IoT of ME and adaptive access for CM service platform. 


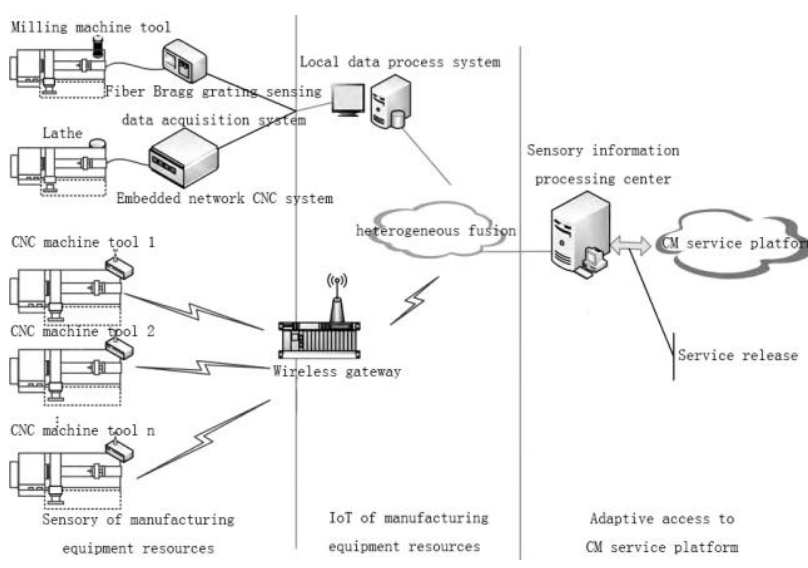

Figure 3. Prototype system of sensory and adaptive access of $\mathrm{CM}$ equipment resources.

\subsection{Sensory of manufacturing equipment resources}

The fiber Bragg grating sensing technology is adopted in sensory of ME resources to realize real-time, dynamic and online sensory and data acquisition of ME running status.

\subsection{Adaptive access and loT of manufacturing equipment resources}

In the adaptive access and IoT of ME resources, the various and polymorphic information obtained through fiber Bragg grating sensing technology and embedded monitoring system have different transmission requirements. For large quantity and high real-time demand information Ethernet is used to be access to the IoT environment, and for small quantity and low realtime demand information RFID is used to be access to the IoT environment. The corresponding type of wireless network is chosen according to the deployment and location of ME in workshop.

\subsection{Adaptive access of manufacturing equipment resources for $\mathrm{CM}$ service platform}

The adaptive access prototype system of $\mathrm{CM}$ equipment resources is composed of the release module and remote invocation module of CM resources service. The acquired sensory data are accessed by release module to go through intelligent analysis, preprocess and storage. And according to the demand, the processing ability of ME is encapsulated into corresponding service to release and then the service is remotely invocated through remote invocation module. The release and invocation modules are developed by SSH (struts + spring + hibernate) framework and Web Service technology is integrated in the modules. The release and invocation architecture of $\mathrm{CM}$ equipment resources service is shown in figure 4 .

Firstly the status and ability of ME resources are virtualized and then are released in the form of service. The service encapsulated in the prototype system mainly include real-time dynamic displayed ME running status data and data analysis results. The system will encapsulate the real-time parameter data of the ME running status and service into WSDL (Web Service Description Language) to release on the server, thus providing interface for remote invocation server. Then the remote invocation module which is built through $\mathrm{B} / \mathrm{S}$ (Browser/Server) method can quickly invocate the service without restriction of region and time.

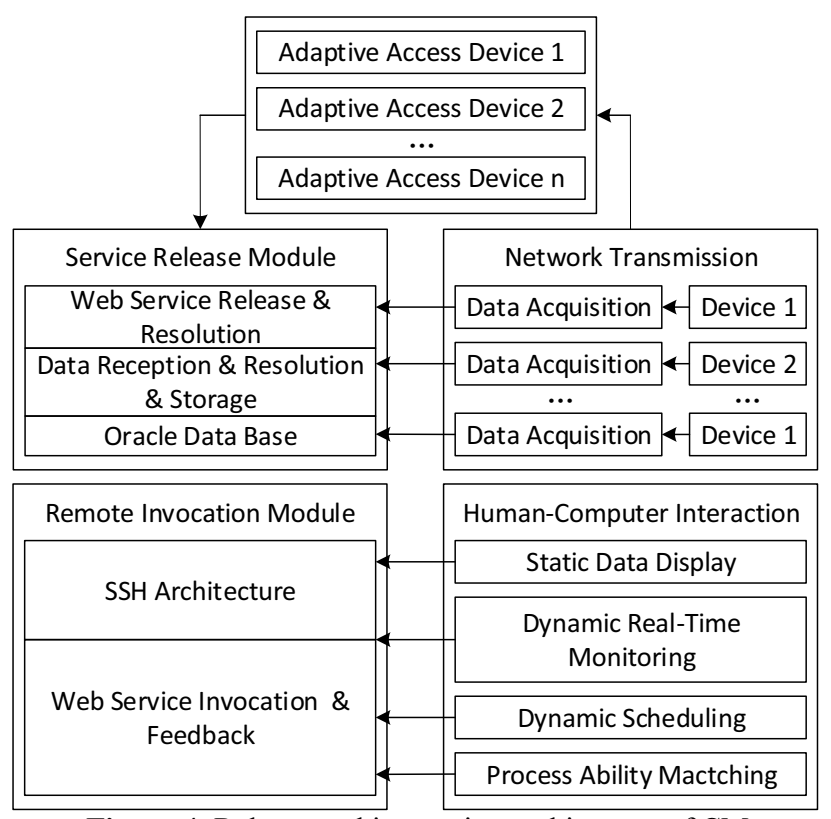

Figure 4. Release and invocation architecture of CM equipment resources service.

\section{Conclusion}

From the sensory, IoT and adaptive access for CM service platform, this paper has discussed the sensory technology and adaptive access technology of $\mathrm{CM}$ equipment resources. The characteristics and key technology of sensory of ME are discussed and the importance and main research contents of fiber Bragg grating sensing technology for sensory of ME resources running status are analyzed. The heterogeneous fusion architecture implementing wide IoT of ME resources is discussed. Various methods and devices heterogeneously and seamlessly access to IoT environment are put forward. From the aspects of information fusion, description and retrieval, and service release and invocation, the adaptive access of multi-source, large quantity and real-time dynamic sensory information of ME for CM service platform is studied. Finally, through some prototype system applications of intelligent sensory and adaptive access for typical ME resources in CM, the related key technologies, implementation methods and devices proposed in this paper are illustrated and described in detail.

\section{References}

1. Buyya R, Yeo C S, Venugopal S, et al. Cloud computing and emerging IT platforms: Vision, hype, and reality for delivering computing as the 5th 
utility[J]. Future Generation computer systems, 2009, 25(6): 599-616.

2. Li B H, Zhang L, Ren L, et al. Further discussion on cloud manufacturing[J]. Computer Integrated Manufacturing Systems, 2011, 17(3): 449-457(in Chinese).

3. Ren L, Zhang L, Zhang Y B, et al. Resource virtualization in cloud manufacturing $[\mathrm{J}]$. Computer Integrated Manufacturing Systems, 2011, 17(3): 511518(in Chinese).

4. Zhou Z, Liu Q, Xu W. From digital manufacturing to cloud manufacturing $[\mathrm{J}]$. Int J Eng Innov Manag, 2011, 1(1): 1-14.

5. Wood K, Brown T, Rogowski R, et al. Fiber optic sensors for health monitoring of morphing airframes: I. Bragg grating strain and temperature sensor[J]. Smart Materials and structures, 2000, 9(2): 163.

6. Gubbi J, Buyya R, Marusic S, et al. Internet of Things (IoT): A vision, architectural elements, and future directions[J]. Future Generation Computer Systems, 2013, 29(7): 1645-1660.

7. Atzori L, Iera A, Morabito G. The internet of things: A survey[J]. Computer networks, 2010, 54(15): 2787-2805.

8. Wang Xiaoyan. The research on the cooperation technology of the wireless heterogeneous network[D]. Wuhan Huangzhong University of Science and Technology, 2006(in Chinese).

9. Zhang L, Luo Y L, Tao F, et al. Key technologies for the construction of manufacturing cloud[J]. Computer Integrated Manufacturing Systems, 2010, 16(11): 2510-2520(in Chinese).

10. Singh S, Choi K, Kodali A, et al. Dynamic fusion of classifiers for fault diagnosis[C]//SMC. 2007: 24672472.

11. Tao F, Hu Y, Zhao D, et al. Study on manufacturing grid resource service QoS modeling and evaluation[J]. The International Journal of Advanced Manufacturing Technology, 2009, 41(9-10): 10341042. 